\title{
An enzyme to cure age-related diseases
}

\author{
More than 35 years ago, telomerase activity was discovered by Elizabeth H. Blackburn and Carol W. Greider. Today, \\ this enzyme is a promising approach to curing some age-related diseases as well as cancer, but it took time for \\ telomerase to be in the spotlight.
}

\section{Paula Martinez and Maria A. Blasco}

T he existence of an enzyme, a DNA polymerase, that could elongate de novo the ends of chromosomes or telomeres was predicted in 1984 on the basis of telomere maintenance and net telomere elongation in both ciliates and yeast ${ }^{1}$. Some fifty years before that discovery, the work of Hermann Müller and Barbara McClintock had showed the occurrence of a special structure at chromosome ends - which Müller termed the telomere - that was essential to prevent chromosomes from fusing to each other ${ }^{2,3}$. The subsequent elucidation of the structure of the DNA molecule and the understanding of the DNA replication mechanisms posed the question on how to maintain the ends of chromosomes throughout cellular and organismal generations. James D. Watson and Alexey M. Olovnikov had envisioned that the ends of linear DNA molecules, such as chromosomes, would shorten every time a cell divides, owing to the incomplete replication of the ends by the conventional polymerases, a problem termed the end replication problem. Thus, to allow for species maintenance, an enzyme must exist that would compensate for the ever-shorter telomeres associated to cell division. Such an enzyme was predicted by Elizabeth H. Blackburn and Jack W. Szostak to elongate the $3^{\prime}$ ends of telomeric repeats to compensate for telomere loss associated to cell division, so that in this manner the ends of chromosomes would not wear away after a few cell divisions, generating chromosomal instability.

To find such an enzyme was of potential great importance, as its telomere elongating activity or the lack thereof could explain the limited life span of normal cells, also known as the Hayflick limit ${ }^{4}$ as well as organismal
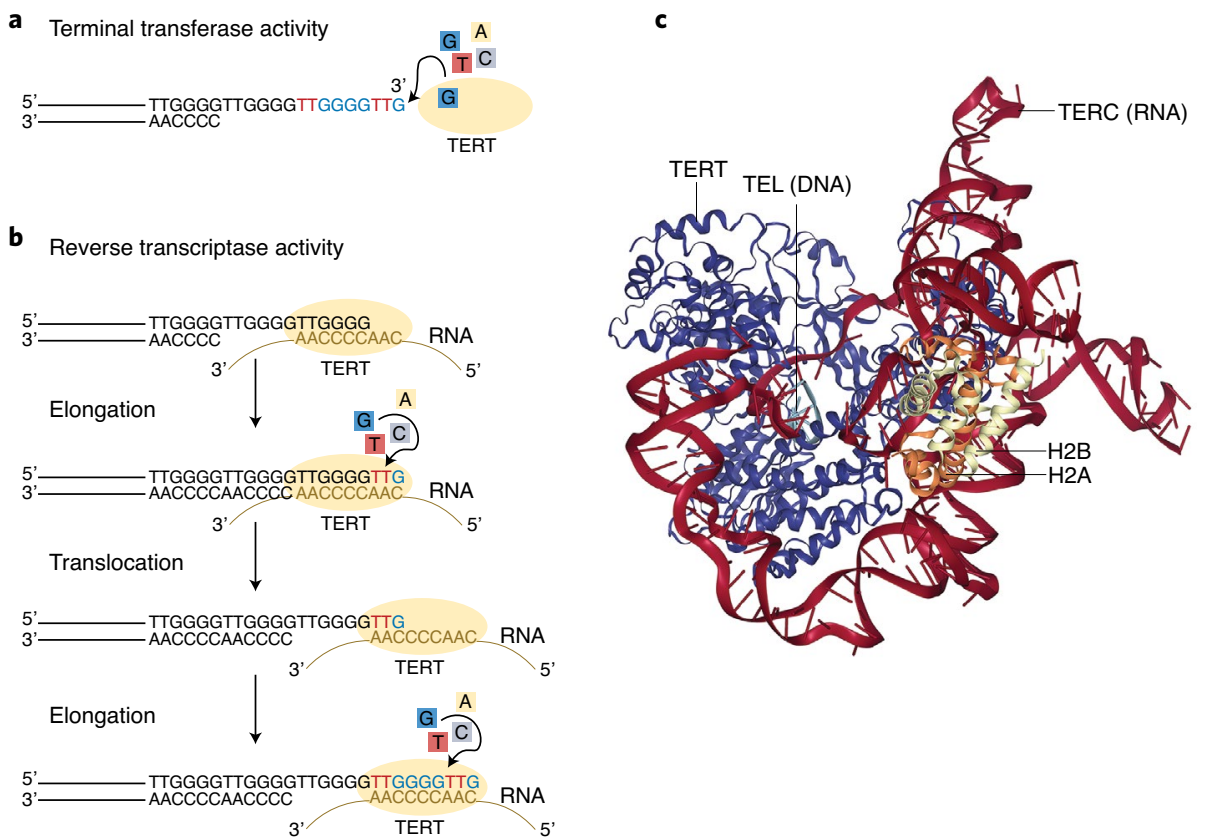

Fig. 1 | Models for telomere elongation by telomerase. a, Telomerase as an enzyme with nucleotide terminal transferase activity was proposed by Greider

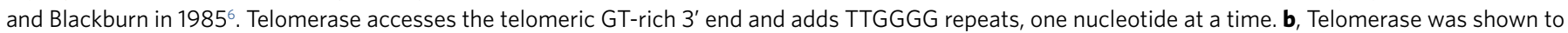

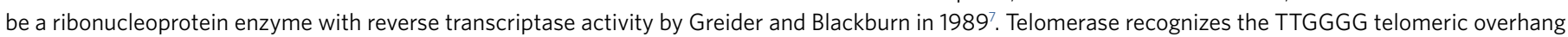
and the CAACCCCAA sequence in the RNA telomerase component hybridizes with it, leaving the last three nucleotides in the RNA unpaired and serving as the template for TTG synthesis. Telomerase is then translocated so that the newly synthesized TTG nucleotides hybridize with the RNA component. A new

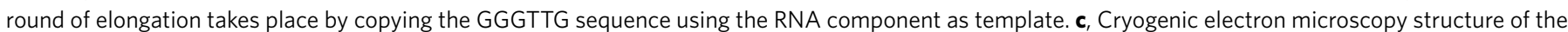
catalytic core of human telomerase (TERT and TERC) in complex with a telomeric DNA substrate and histones H2B and H2A. The structure reveals DNA-

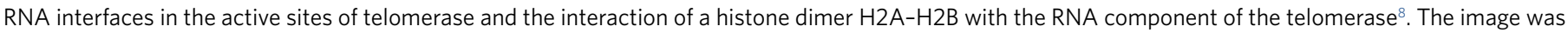
created with the PDB under accession number 7BG9 and NGL viewer ${ }^{8,14}$. 
aging and longevity. Such an activity could also explain the immortal nature of cancer cells and their ability to divide indefinitely.

While working at Joe Gall's group, Blackburn had sanger-sequenced the first chromosome end from the ciliate Tetrahymena and found a gene-less repeated sequence heterogeneous in length ${ }^{5}$. Possibly, the fact that the ends of chromosomes did not harbour any gene that could explain their function decreased the enthusiasm of the scientific community but not that of Blackburn, who was convinced that such repeated DNA must somehow hold the key to aging and longevity of life.

Elizabeth H. Blackburn and a new $\mathrm{PhD}$ student in her lab, Carol W. Greider, set out to find such an enzyme responsible for telomere lengthening. In 1985, they eventually observed activity for the synthesis of telomeric repeats de novo in Tetrahymena cell-free extracts ${ }^{6}$. In that work, they designed an elegant in vitro assay using cell-free Tetrahymena extracts as a source of telomere elongation activity, a single strand DNA oligomer (TTGGGG) as a substrate for the reaction and different combinations of radioactively labelled and unlabelled nucleoside tri-phosphate. They found a clear six-base repetition signature in the autographs of polyacrylamide gels that was only detected in the presence of either $\alpha^{-32}$-P-dGTP and dTTP or ${ }^{32}$-P-dTTP and $\mathrm{dGTP}$, indicating that the terminal synthesis consisted of TG-containing repeats. To ultimately probe that only TTGGGG repeats were synthesized, dideoxynucleotides that are DNA chain-elongation inhibitors were added to the reaction mix and they observed that only ddTTP and ddGTP, and not ddATP or ddCTP, inhibited the reaction. Addition to the reaction mix of either micrococcal nuclease or the $\alpha$ DNA polymerase inhibitor aphidicolin had no effect on this repeat addition activity, demonstrating that the activity was independent of both endogenous Tetrahymena DNA and DNA polymerases. That this activity was endowed to an enzyme was supported by the fact that heating the extract to $90^{\circ} \mathrm{C}$ or treatment with proteinases fully blocked repeat addition. The presence of the telomeric oligomer single strand primers specific for both Tetrahymena's and yeast's sequences were functional for priming the reaction while the complementary telomeric (CCCCAA) or other non-telomeric sequences were not. Nevertheless, the researchers speculated that the elongation activity was template-independent and explained that the specificity of the reaction for only the GT-rich strand must be conferred by higher-order structural features adopted by these sequences. They concluded that it must be a telomere-specific terminal transferase based on the fact that a DNA template was not needed to generated the ladder of telomeric TTGGGG repeats (Fig. 1a). They did not contemplate, however, that the telomere elongating enzyme could use an RNA component as template complementary to the single strand oligomer primer, thus conferring the observed primer sequence specificity ${ }^{6}$. Had they used different GT-rich sequences as primers, other than those of Tetrahymena and yeast, and added ribonuclease to the reaction, they would have realized at the time the requirement of a specific RNA sequence template. Indeed, soon they discovered that telomerase had an associated RNA component that was used as a template for the addition of repeats ${ }^{7}$. Later on, Blackburn discovered that the protein component of telomerase was indeed a reverse transcriptase (Fig. 1b). In the following years up to present, intensive and fruitful research has been performed in the field, culminating recently in the resolution by cryogenic electron microscopy of the structure of human telomerase bound to telomeric DNA at sub-4- $\AA$ resolution ${ }^{8}$ (Fig. 1c).

Tetrahymena is a ciliate with a peculiar life cycle and thus it remained unclear whether its mechanisms of telomere maintenance would be universal, making it urgent to find out whether similar activities and mechanisms existed in other organisms and in particular, in mammals. In 1990, Calvin Harley and Carol W. Greider demonstrated that telomeres were indeed shortening with age in humans, presumably as the consequence of telomerase deficiency in adult cells, a phenomenon that could explain aging . Final demonstration of the importance of telomerase to maintain mammalian telomeres came from the generation of the first mice deficient in a previously identified putative telomerase RNA component. These mice had shorter telomeres than normal and a higher chromosomal instability, as predicted by Müller and McClintock, as well as faster aging and less cancer ${ }^{10}$. Later on, humans carrying mutations in the telomerase genes were shown to have a number of diseases known as telomere syndromes ${ }^{11}$, which recapitulated the phenotypes of telomerase-deficient mice, demonstrating that telomere length also plays a central role for aging in humans. But not only in humans; recent work has shown that the rate of telomere shortening seems to be a universal trait that can explain species longevity ${ }^{12}$.

Not only telomere syndromes but also some age-related diseases have been associated to the presence of short telomeres, suggesting that telomerase activation could be a potential therapeutic strategy to prevent or treat several diseases. In this regard, gene therapy vectors carrying the mouse telomerase TERT gene have been shown to be effective in the treatment of mouse models of diseases associated to short telomeres, such as pulmonary fibrosis, heart infarction and aplastic anemia ${ }^{13}$. Pulmonary fibrosis is a particularly attractive indication for telomerase activation strategies as it is a lethal disease with a current lack of effective treatments. Furthermore, it is a frequent sequel in COVID-19 patients.

Future clinical trials with telomerase activation strategies for the treatment of pathologies associated to short telomeres, such as some cases of pulmonary fibrosis, are not unlikely in a near future. Should these treatments work, it would be the first time that an age-associated disease is effectively treated based on the deep knowledge of the molecular biology of aging mechanisms elucidated based on the work by Elizabeth H. Blackburn and Carol W. Greider.

\section{Paula Martinez and Maria A. Blasco (D) 凶 Telomeres and Telomerase Group, Molecular Oncology Program, Spanish National Cancer Centre (CNIO), Madrid, Spain. \\ $\bigotimes_{e-m a i l: m b l a s c o @ c n i o . e s}$}

Published online: 21 September 2021 https://doi.org/10.1038/s41929-021-00677-z

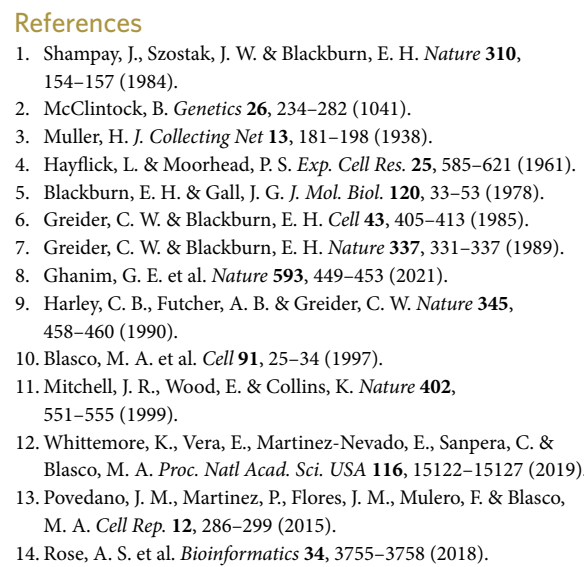

Competing interests

The authors declare no competing interests. 